\title{
Estudio de aleaciones con alto contenido en níquel procesadas mediante manufactura aditiva
}

\section{Research on high nickel content alloys processed by additive manufacturing}

Presentación: 22/11/2019

\section{Doctorando:}

\section{Pedro Martín Lerman}

Universidad Tecnológica Nacional, Facultad Regional Córdoba

pedro.lerman@ar.mahle.com

\section{Director/es:}

\section{Mirco Chapetti \\ Roberto Lucci}

\section{Resumen}

La manufactura aditiva es una técnica de fabricación en franca expansión. Naturalmente y como cualquier otro proceso productivo, su aplicabilidad posee ciertas limitaciones. Sin embargo, aún existen gaps de factibilidad que podrían ser superados como por ejemplo aquellos presentes en sectores productivos de alto volumen y bajos márgenes de rentabilidad. La presente investigación intenta generar un aporte incremental que permita expandir sus posibilidades de aplicación, especialmente cuando se consideran componentes de alta criticidad y exigencia termomecánica. Para ello, dos objetivos clave serán considerados, el desarrollo de un material de relación costoperformance superior a los ya existentes y la optimización en su productividad respecto al estado del arte.

En esta publicación se detallan las actividades delineadas a fin de desarrollar los parámetros específicos de procesamiento de la aleación NCF 3015 mediante técnicas de manufactura aditiva del tipo "Fusión Selectiva por Láser" (SLM).

Palabras claves: Manufactura aditiva, aleaciones intermedias, NCF 3015, reducción de costos.

\section{Abstract}

Additive manufacturing is a frankly expanding manufacturing technique. Like any other productive process, its applicability has certain limitations. However, there are still feasibility gaps that could be overcome like those found in high volume - low profit margin productive fields. This research intends to provide an incremental contribution which would lead to expand its application possibilities. In this sense, two key objectives will be considered, the development of a cost-effective material suitable to be processed by additive manufacturing and the productiveness optimization with respect to the state of the art.

This publication details the outlined activities intended to develop specific process parameters to additive manufacture the alloy NCF 3015 by means of "Selective Laser Melting (SLM)"

Keywords: Additive manufacturing, intermediate Nickel alloys, NCF 3015, cost reduction.

\section{Introducción}

La manufactura aditiva es una técnica de producción innovadora, de carácter disruptivo, que consiste en la fabricación de componentes partiendo de materias primas en forma de polvo, alambre o láminas, los cuales son 
consolidados a través de un proceso que se gesta capa a capa [1-2]. Al compararla con las técnicas convencionales de fabricación, puede decirse que prácticamente no existen limitaciones de diseño, posee una eficiencia de material cercana al 100\%, los post procesamientos son mínimos y el tiempo de espera entre pedido y recepción es prácticamente despreciable. Sin embargo, no hay razón alguna para pensar en un reemplazo universal de los métodos productivos tradicionales. Por ejemplo, dentro del campo de los componentes metálicos estructurales e independientemente de su extraordinaria potencialidad, existen todavía algunas limitaciones frente a otras técnicas de manufactura lo cual restringe la ampliación de sus fronteras. Ejemplos de ello son los elevados costos de producción, el máximo tamaño de pieza alcanzable y las inferiores propiedades mecánicas frente a los productos forjados. Debido a ello, el crecimiento exponencial registrado en mercados específicos tales como el aeroespacial o el medicinal no ha podido ser extrapolado a la industria automotriz; transformar a este nuevo pilar en un verdadero jugador clave requiere de drásticas optimizaciones tecnológicas no resueltas hasta el momento.

La presente investigación pretende aportar conocimientos específicos sobre dos de las principales restricciones que enfrenta la manufactura aditiva para lograr una franca irrupción en la industria automotriz, el desarrollo de nuevas aleaciones de relación costo-beneficio superior y el aumento en la velocidad de deposición o productividad. Se focalizará en aleaciones de elevadas propiedades termomecánicas como lo es el NCF 3015, un acero de características cercanas a las aleaciones base níquel tradicionales, y en la optimización de las estrategias y técnicas de impresión para la reducción de los tiempos de ciclo sin actuar en detrimento de las propiedades funcionales finales.

\section{Resultados}

Pocos materiales han sido desarrollados para ser producidos mediante manufactura aditiva. Sería de esperarse que incrementar el abanico de posibilidades conllevaría a elevadas inversiones en I+D que no todas las compañías están posibilitadas a afrontar. Sin embargo, una visión en perspectiva de la problemática podría suponer que tales esfuerzos podrían no ser tan prohibitivos. Por ejemplo, sería factible asumir que para una familia de aleaciones, sus mecanismos metalúrgicos de fusión y solidificación responderían de manera similar. Esto implicaría que, si existiera alguna aleación ya desarrollada, parte del camino a recorrer por sus pares se encontraría develado. Por otro lado, existe una amplia referencia bibliográfica sobre innumerables aleaciones forjadas o coladas que si fuera extrapolada a la técnica de manufactura aditiva permitiría inferir un nivel aproximado en sus propiedades mecánicas finales.

Como punto de partida, considérese el escenario de componentes mecánicos que operan en ambientes muy exigentes tales como los descriptos en la Tabla $\mathrm{N}^{\circ} 1$ :

Tabla $\mathbf{N}^{\circ}$ 1: Condiciones funcionales de referencia

\begin{tabular}{|l|l|}
\hline Temperatura & $>750^{\circ} \mathrm{C}$ \\
\hline Esfuerzos & Dinámicos $>170 \mathrm{MPa} \times 1 \mathrm{e}^{7}$ mínimo \\
\hline Ambiente & Oxidativo, corrosión en caliente y por condensados, migración y pérdida de elementos de aleación \\
\hline
\end{tabular}

La aleación Inconel 718 es el material base níquel de referencia para impresión 3D y su efectividad para satisfacer estas demandas operativas está prácticamente asegurada. No obstante, el costo de este material en combinación con la las técnicas de impresión 3D excede las posibilidades de sustitución de gran parte de los componentes fabricados por las vías tradicionales. Por el contrario, otros materiales de menor costo y también disponibles para ser procesados mediante manufactura aditiva no logran cumplir con los requisitos funcionales mencionados con anterioridad.

En los últimos años, la industria automotriz ha desarrollado una gama de materiales de alta performance y bajo costo capaces de operar bajo las condiciones funcionales referenciadas. Estas aleaciones, que se ubican entre los aceros inoxidables y las aleaciones base níquel, son conocidas como Aleaciones Intermedias en Níquel o Aceros de alto Níquel. Un ejemplo de ellas es la aleación NCF 3015 [3-4] donde su contenido de este elemento es aproximadamente un 50\% menor que el de sus pares base níquel. La tabla $\mathrm{N}^{\circ} 2$ muestra una comparación entre composiciones químicas nominales de las aleaciones AISI 316, NCF 3015 e Inconel 718.

Dado que no existe plan alguno que considere la provisión seriada de este material para ser procesado mediante manufactura aditiva, por el momento es muy dificultoso lograr estimar un precio base de referencia. Los proveedores de materias primas, al restringir sus opciones de fabricación a reactores de laboratorio en instalaciones de $\mathrm{I}+\mathrm{D}$, solo pueden informar costos a nivel piloto los cuales no reflejan su verdadero potencial. Es posible, sin embargo, reflejar de forma estimativa la posición de esta aleación frente a otros materiales considerando valores disponibles para productos largos laminados. Esta comparación puede observarse en la tabla $\mathrm{N}^{\circ} 3$ mientras que la tabla $\mathrm{N}^{\circ} 4$ informa precios de referencia de 
distintas aleaciones empleadas en manufactura aditiva y los valores obtenidos para la producción de $100 \mathrm{Kg}$ de NCF 3015 a nivel piloto.

Tabla $\mathrm{N}^{\circ}$ 2: Composición química nominal [\%], sin impurezas ni trazas.

\begin{tabular}{|c|c|c|c|}
\hline Elemento Aleación & AISI 316L & NCF 3015 & Inconel 718 \\
\hline $\mathrm{C}$ & 0.03 máx. & 0,08 máx. & 0,08 máx. \\
\hline $\mathrm{Si}$ & 0.5 & 0,5 máx. & 0.35 \\
\hline $\mathrm{Mn}$ & 1 & 0,5 máx. & 0.35 \\
\hline $\mathrm{Cr}$ & 17.5 & 14,5 & 52.5 \\
\hline $\mathrm{Ni}$ & 11.5 & 31,5 & 5.1 \\
\hline $\mathrm{Nb} \mathrm{Ta}$ & & 0,7 & 3.05 \\
\hline $\mathrm{Mo}$ & 2.25 & 0,75 & 0.9 \\
\hline $\mathrm{Co}$ & & 2,6 & 0.5 \\
\hline $\mathrm{Ti}$ & & 1,9 & Balance \\
\hline $\mathrm{Al}$ & Balance & & \\
\hline $\mathrm{Fe}$ & & & \\
\hline Valores expresados en porcentaje en peso. & & \\
\hline
\end{tabular}

Tabla $\mathbf{N}^{\circ}$ 3: Precio de material por $\mathrm{Kg}$ [USD/Kg] en productos largos laminados, solubilizados y rectificados - diámetro $10.5 \mathrm{~mm}$

\begin{tabular}{|l|l|}
\hline NCF 3015 & $17,92^{2}$ \\
\hline Nimonic $80 \mathrm{~A}^{1}[5]$ & $27,57^{2}$ \\
\hline 1. & Nimonic $80 \mathrm{~A}$ es una aleación base níquel de referencia que puede compararse con el Inconel 718. \\
2. & Precios de referencia: Julio 2019, mismo proveedor; datos del proveedor en reserva. \\
\hline
\end{tabular}

Tabla $\mathbf{N}^{\circ}$ 4: Precio de material [USD/Kg] para manufactura aditiva / SLM / 15-45 $\mu \mathrm{m}$

\begin{tabular}{|l|l|}
\hline $316 \mathrm{~L}^{1}$ & 60 \\
\hline Inconel $718^{1}$ & 100 \\
\hline NCF 3015 & 390 \\
\hline NCF 3015 & 202 \\
\hline 1. & Proveedor A \\
2. & Proveedor A. 2 coladas de atomización, base mínima de compra: $80 \mathrm{Kg}$. \\
3. & Proveedor B. Base mínima de compra: $100 \mathrm{Kg}$ \\
\hline
\end{tabular}

Los valores de referencia de los productos forjados indican que la aleación NCF 3015 tiene un costo 35\% inferior al de las súper aleaciones base níquel. Por el contrario, al no estar desarrollada para ser procesada mediante manufactura aditiva, su costo aparece entre un 100 y $400 \%$ superior a las mismas.

Distinto es el caso de las propiedades físicas, químicas y mecánicas donde se dispone de gran variedad de datos comparativos que respaldan su selección. A nivel informativo, la figura $\mathrm{N}^{\circ} 1$ muestra datos comparativos de dureza y resistencia a la tracción, ambos en función de la temperatura.

El avance inicial sobre la propuesta doctoral ha implicado atravesar un camino tortuoso, principalmente debido a los elevados costos de fabricación. Sin embargo, se han logrado alcanzar hitos claves y es de suponerse que los grandes obstáculos ya han sido superados. Dos de los más importantes fueron la selección final del proveedor y la compra del material el cual estará disponible a partir de Febrero de 2020. En paralelo y luego de una exhaustiva revisión de los potenciales centros de impresión, se ha logrado seleccionar a aquél que mejor se adapta a las necesidades y posibilidades de esta investigación. A continuación se brindan detalles sobre los avances logrados durante el período 2019. La figura №2 muestra las actividades troncales descritas en línea temporal.

En referencia a los centros de impresión, la selección se orientó al único proveedor local disponible hasta el momento, CENTEC / INTI Rafaela, el cual cuenta con un equipo de última generación EOS 290 M, capacidad, flexibilidad operativa y costos de servicios acordes al presupuesto disponible. Cabe mencionar que otras opciones en el exterior implicaban erogaciones en el rango de 50.000 a 100.000 dólares. 

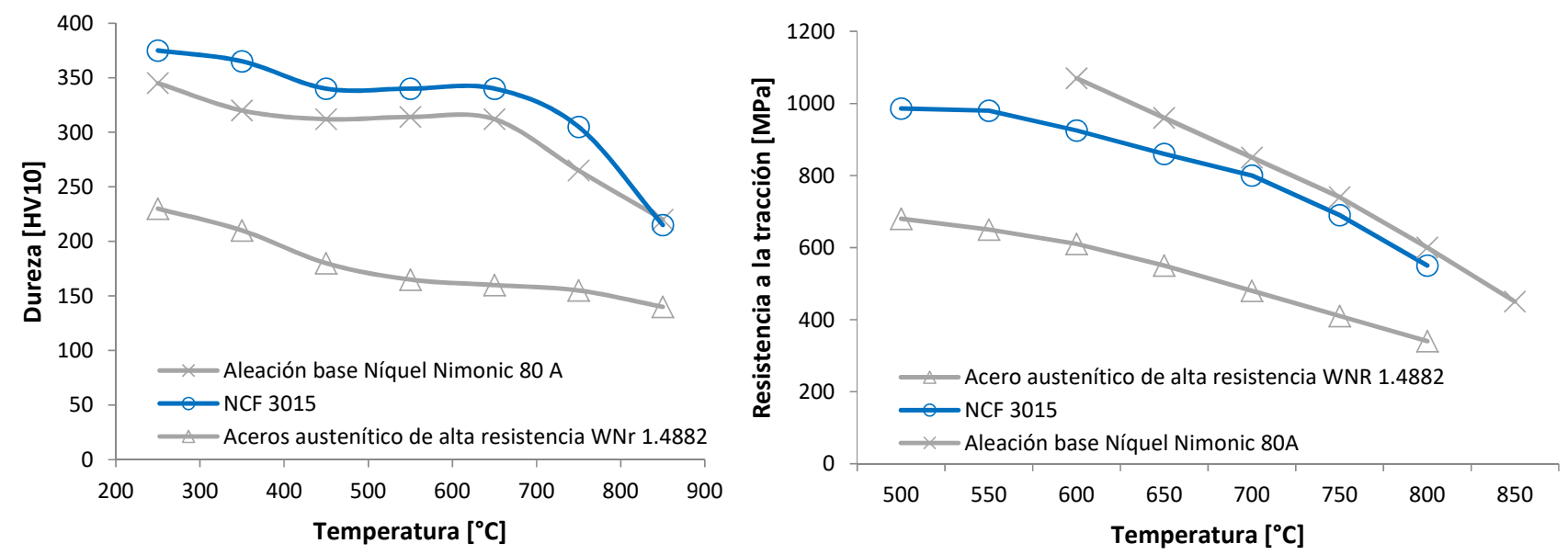

Figura $\mathrm{N}^{\circ}$ 1: Dureza y resistencia a la tracción en función de la temperatura para una aleación base níquel, un acero de alta resistencia y un material intermedio, NCF 3015.
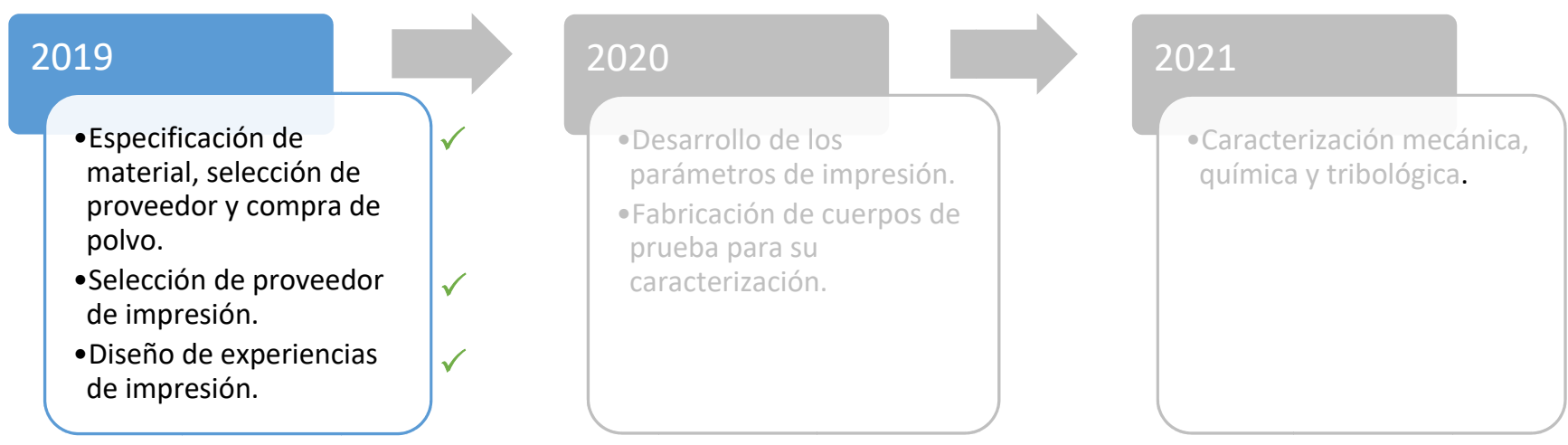

Figura $\mathrm{N}^{\circ}$ 2: Plan de trabajo a corto y mediano plazo.

El diseño de una receta de impresión adecuada y su consiguiente validación requiere de varias etapas experimentales que parten desde la fabricación de cordones simples hasta la obtención de cuerpos de prueba específicos. La figuras $\mathrm{N}^{\circ} 3$ a 6 indican la metodología de trabajo a emplear mientras que la tabla $\mathrm{N}^{\circ} 5$ resume los ensayos de validación de concepto para cada una de las fases detalladas.

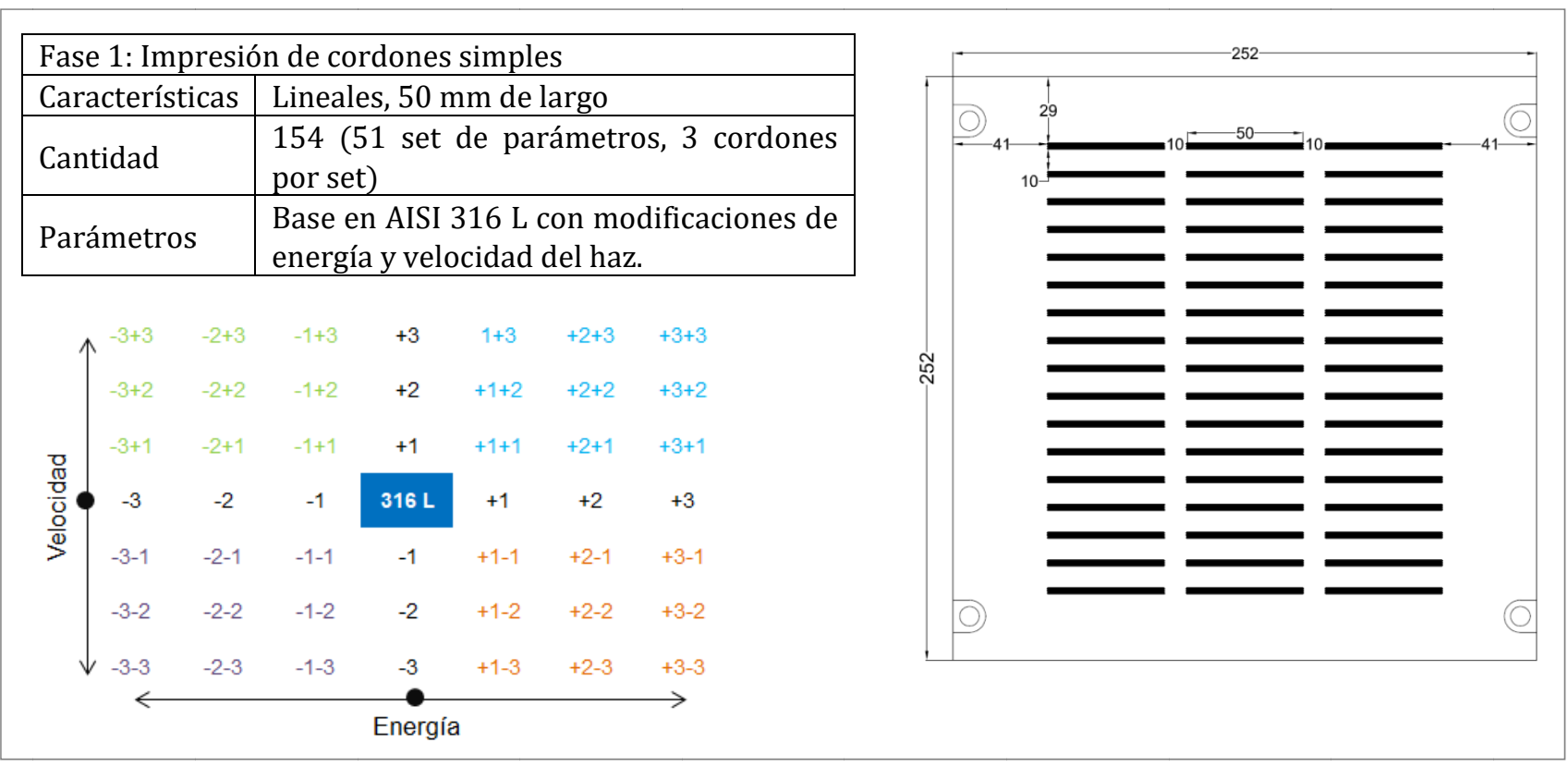

Figura $N^{\circ}$ 3: Fase 1, DoE de parámetros a través de cordones simples de soldadura. 


\begin{tabular}{|l|l|}
\hline \multicolumn{2}{|l|}{ Fase 2: Impresión de monocapas } \\
\hline Características & Superficies cuadradas de $30 \times 30 \mathrm{~mm}$ \\
\hline Cantidad & $\begin{array}{l}45 \quad(5 \text { parámetros iniciales }+2 \\
\text { variaciones de hatch, 3 superficies por } \\
\text { combinación) }\end{array}$ \\
\hline Parámetros & Estrategia lineal unidireccional \\
\hline
\end{tabular}

\begin{tabular}{|c|c|c|c|c|}
\hline $\begin{array}{c}P 1 \\
H+20 \%\end{array}$ & $\begin{array}{c}\mathrm{P} 2 \\
\mathrm{H}+20 \%\end{array}$ & $\begin{array}{c}\text { P3 } \\
H+20 \%\end{array}$ & $\begin{array}{c}\mathrm{P} 4 \\
\mathrm{H}+20 \%\end{array}$ & $\begin{array}{c}\mathrm{P} 5 \\
\mathrm{H}+20 \%\end{array}$ \\
\hline P1 & P2 & P3 & P4 & P5 \\
\hline \multicolumn{5}{|c|}{$\begin{array}{c}\left.\text { Densidad de Energía [W/cm }{ }^{2}\right] \\
\mathrm{P} 1<\mathrm{P} 2<\mathrm{P} 3<\mathrm{P} 4<\mathrm{P} 5\end{array}$} \\
\hline $\begin{array}{c}\text { P1 } \\
\text { H }-20 \%\end{array}$ & $\begin{array}{c}\text { P2 } \\
H-20 \%\end{array}$ & $\begin{array}{c}\text { P3 } \\
\mathrm{H}-20 \%\end{array}$ & $\begin{array}{c}\mathrm{P} 4 \\
\mathrm{H}-20 \%\end{array}$ & $\begin{array}{c}\text { P5 } \\
\text { H }-20 \%\end{array}$ \\
\hline
\end{tabular}

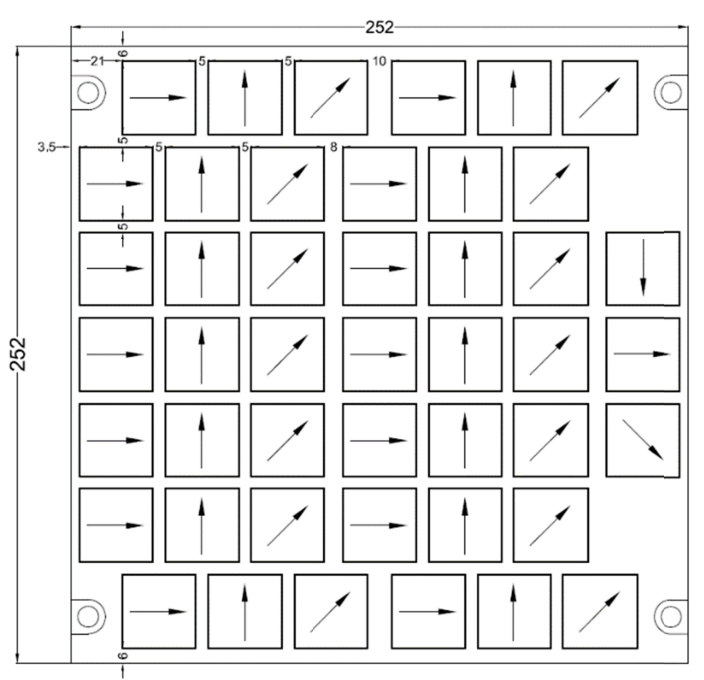

Figura $\mathrm{N}^{\circ} 4$ : Fase 2, DoE de parámetros a través de monocapas de soldadura.

\begin{tabular}{|l|l|}
\hline \multicolumn{2}{|l|}{ Fase 3: Impresión de multicapas } \\
\hline Características & Cuerpos de prueba de 0,8 x 0,8 × 50 mm y $8 \mathrm{~mm}$ de diámetro x 50 mm de altura. \\
\hline Cantidad & $\begin{array}{l}72 \text { (Por cada morfología, } 3 \text { parámetros iniciales, } 4 \text { estrategias de fabricación, } 3 \text { probetas } \\
\text { por cada estrategia). }\end{array}$ \\
\hline Parámetros & $\begin{array}{l}\text { Lineal unidireccional, bidireccional y punto a punto con ángulo de } 67^{\circ} \text { entre capas. } \\
\text { Lineal de relleno de circuito inverso capa a capa. }\end{array}$ \\
\hline
\end{tabular}
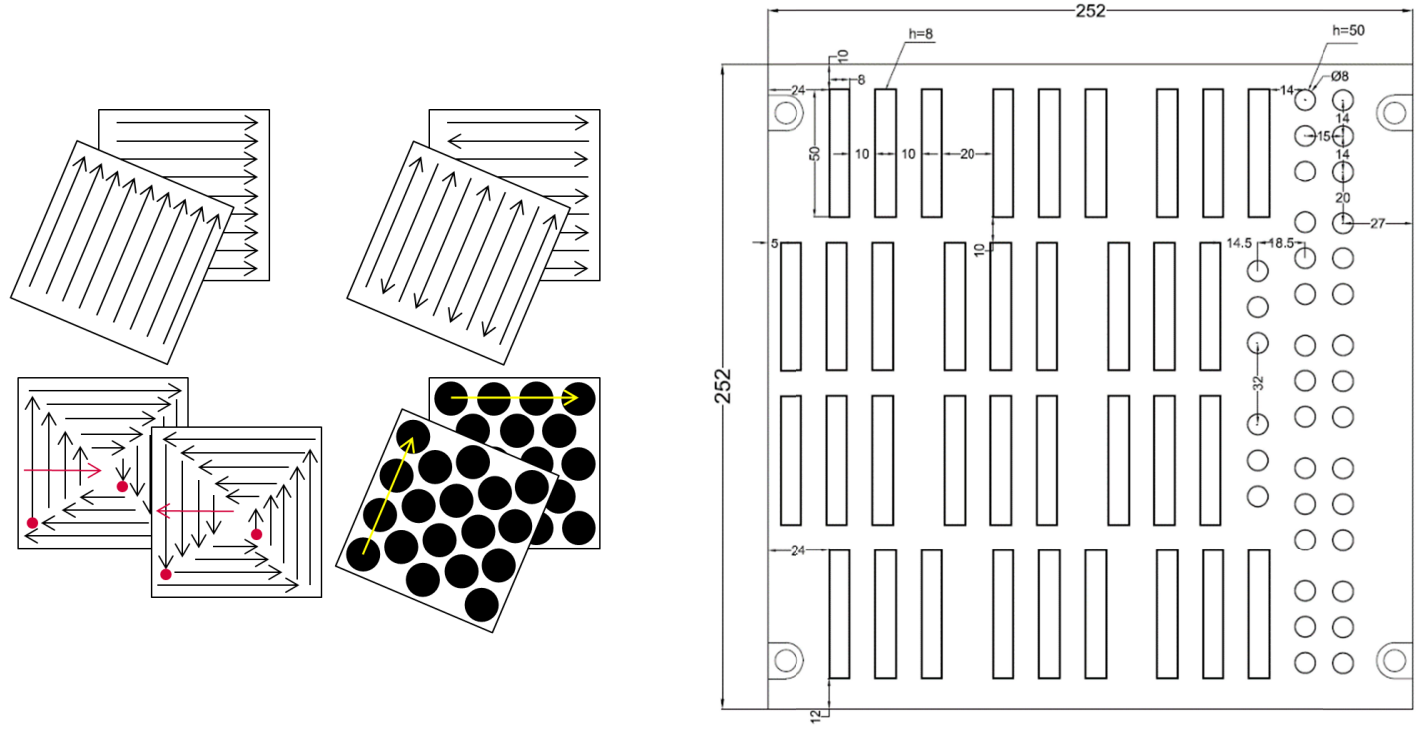

Figura $\mathrm{N}^{\circ}$ 5: Fase 3, DoE de parámetros a través de deposición multicapa de soldadura. 


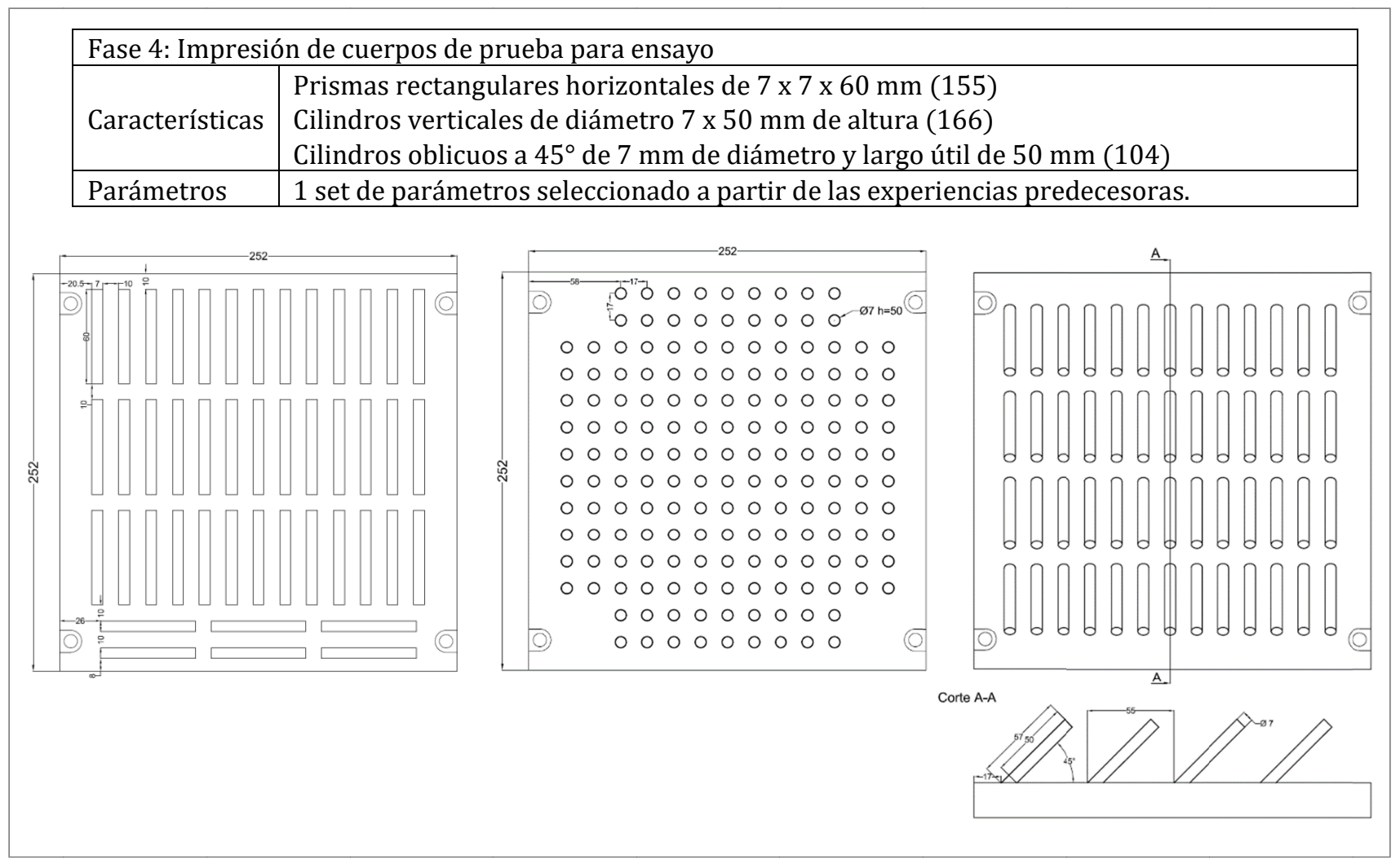

Figura $\mathrm{N}^{\circ}$ 6: Fabricación de cuerpos de prueba para caracterización mecánica, química y tribológica.

Tabla $N^{\circ}$ 5: Ensayos definidos por fase

\begin{tabular}{|l|l|}
\hline $\begin{array}{l}\text { Fase 1 - cordones } \\
\text { simples }\end{array}$ & $\begin{array}{l}\text { Análisis visual, estereoscópico y microscópico. Análisis metalúrgico en secciones transversales } \\
\text { (morfología, penetración, dilución, adherencia). Selección de los mejores parámetros y cálculos } \\
\text { de overlaps. }\end{array}$ \\
\hline Fase 2 - monocapas & Símil Fase 1. \\
\hline Fase 3 - multicapas & $\begin{array}{l}\text { Símil fase 2 más estudios de densidad por tomografía, cuantificación y clasificación de defectos, } \\
\text { tensiones residuales, análisis de dureza, microestructura, caracterización de fases, respuesta a los } \\
\text { tratamientos térmicos y selección de los mejores parámetros de procesamiento para la } \\
\text { caracterización y validación final. }\end{array}$ \\
\hline $\begin{array}{l}\text { Fase } 4-\text { cuerpos de } \\
\text { prueba }\end{array}$ & Símil fase 3 más ensayos de tracción, fatiga, desgaste y corrosión. \\
\hline
\end{tabular}

\section{Referencias}

[1] ISO/ASTM 52900:2015 (en)

[2]ADDITIVE MANUFACTURING, Design, Methods and Processes. Steinar Killi. Pan Stanford Publishing Pte. Ltd. ISBN 978-981-4774-16-1.

[3] Hitachi NCF 3015 Technical Data Sheet.

[4] Villares Metals VAT30 Technical Data Sheet.

[5] Special Metals Nimonic 80A Technical Data Sheet. 